\title{
Detection of enterococcus faecalis in subgingival biofilm of patients with chronic refractory periodontitis
}

\author{
Esrafil Balaei-Gajan ${ }^{1}$, Adileh Shirmohammadi ${ }^{2}$, Rahib Abashov ${ }^{3}$, Mohammad Agazadeh ${ }^{4}$, Masoumeh Fara- \\ marzie $^{5}$
}

${ }^{1}$ Dentist, Department of Community Dentistry, Tabriz Dental Faculty

${ }^{2}$ Associated Professor, Periodontics Department, Tabriz Dental Faculty

${ }^{3}$ Head of Microbiology Department, School of Biology, Baku State University

${ }^{4}$ Assistant Professor, Microbiology Department, Tabriz Medical Faculty

${ }^{5}$ Assistant Professor, Periodontics Department, Tabriz Dental Faculty

Correspondence:

Periodontics Department

Tabriz Dental Faculty

Golgasht St. Tabriz

Iran

shirmohamadia@yahoo.com

Received: 11/06/2009

Accepted: 24/12/2009

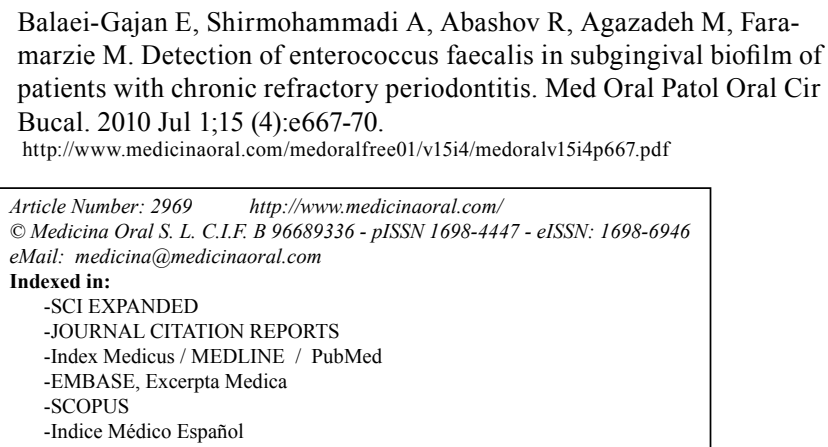

Balaei-Gajan E, Shirmohammadi A, Abashov R, Agazadeh M, Faramarzie M. Detection of enterococcus faecalis in subgingival biofilm of patients with chronic refractory periodontitis. Med Oral Patol Oral Cir Bucal. 2010 Jul 1;15 (4):e667-70.

http://www.medicinaoral.com/medoralfree01/v15i4/medoralv15i4p667.pdf

\begin{abstract}
Objectives: Refractory periodontitis is the occurrence of additional clinical attachment loss after repeated attempts to control the infection with conventional periodontal therapy. Some microorganisms seem to be involved in the pathogenesis of chronic refractory periodontitis. The prevalence of Enterococcus faecalis in the oral cavity seems to be higher in individuals with periodontitis. Therefore, the present study investigated the presence of $\mathrm{E}$. faecalis in subgingival biofilm of patients with chronic refractory periodontal disease.

Study design: Periodontal treatment was instituted for 100 patients suffering from chronic periodontitis. Then samples were obtained from 27 successfully treated and 27 chronic refractory periodontitis subjects and then cultured. Statistical evaluation was performed for descriptive purposes.

Results: $27 \%$ of the patients had chronic refractory periodontitis. The difference in the presence of $\mathrm{E}$. faecalis in the pockets between the successfully treated $(11.1 \%)$ and chronic refractory $(51.8 \%)$ groups by culture methods was statistically significant $(\mathrm{P}<0.05)$.

Conclusion: Data showed that E. faecalis is probably involved in the pathogenesis of refractory periodontitis. Accurate knowledge about the pathogen and its role in the pathogenesis of refractory infections helps develop effective treating strategies.
\end{abstract}

Key words: Enterococcus faecalis, periodontitis, refractory, bacteria. 


\section{Introduction}

Enterococci are normal human commensals adapted to the nutrient-rich, oxygen-depleted, and ecologically complex environments of the oral cavity, gastrointestinal tract, and vaginal vault (1). Enterococci now rank among the top three nosocomial bacterial pathogens and strains resistant to currently available antibiotics and pose real therapeutic difficulties. Up to $90 \%$ of enterococcal infections in humans are caused by Enterococcus faecalis (2).

Data on oral prevalence of E. faecalis vary widely in different studies (3-6). The presence of E. faecalis in the oral cavity raises the question of whether the mouth could be a source for infection with this microorganism. Moreover, it seems conceivable that the oral status influences, directly or indirectly, the colonization by E. faecalis. For instance, enterococci were detected in samples from multiple oral sites in $60 \%$ of school children with high caries activity and $75 \%$ of patients with endodontic infection (4). More recently, Sedgley et al. (7) reported the presence of E. faecalis in $29 \%$ of oral rinse samples, $55 \%$ of tongue dorsum and $22 \%$ of gingival sulcus samples from 41 endodontic subjects. In addition, the microorganism has been reported as the species most commonly recovered from teeth with failed endodontic treatment and persistent infections $(8,9)$. In particular, individuals with periodontal diseases may harbor higher proportions and levels of several bacterial pathogens of medical importance compared to periodontally healthy individuals $(3,10)$. However, only a limited number of studies have evaluated the correlation between the prevalence of E. Faecalis and periodontal diseases $(3,5,10,11)$. The potential pathogenic role of this species in periodontal infections is unknown. Nonetheless, E. faecalis possesses various virulence factors which may contribute to periodontal inflammation and tissue destruction.

In a study carried out by Souto et al. (12) E. faecalis was detected significantly more often in saliva and subgingival samples of periodontitis patients $(40.5 \%$ and $47.8 \%$, respectively) compared to the periodontally healthy group (14.6\% and $17.1 \%$, respectively; $\mathrm{P}<0.05$ ).

Enterococcus faecalis is the most commonly isolated or detected species from oral infections, including marginal periodontitis, infected root canals, and periradicular abscesses (2).

Enterococci are well-adapted for survival and persistence in a variety of adverse environments. It is resistant to the antimicrobial effects of calcium hydroxide, probably partly due to an effective proton pump mechanism which maintains optimal cytoplasmic $\mathrm{pH}$ levels (13). The rapid emergence of antimicrobial resistance among enterococci helps shift the microbial flora in favor of Enterococcus faecalis.

The term refractory periodontitis refers to destructive periodontal diseases in patients who, when longitudinally monitored, demonstrate additional attachment loss at one or more sites, despite well-executed therapeutic and patient efforts to stop the progression of disease. These diseases may occur in situations where conventional therapy has failed to eliminate microbial reservoirs of infection, or has resulted in the emergence or superinfection of opportunistic pathogens. They may also occur as the result of a series of unknown factors which may compromise the host's response to conventional periodontal therapy (14). In view of the limited data, the aim of this study was to determine the occurrence of E. faecalis in subgingival biofilm from successfully treated or chronic refractory periodontitis patients. It is probable that $\mathrm{E}$. faecalis would be a factor in failure of therapy. Therefore, the aim of this study was to evaluate the presence of $\mathrm{E}$. faecalis in chronic refractory periodontitis patients in order to make comparisons with successfully treated chronic periodontitis patients.

\section{Materials and Methods}

A total of 100 non-smoking chronic periodontitis patients aged 30-50 years old referring to the Periodontics Department at Tabriz University of Medical Sciences School of Dentistry, Tabriz, Iran, with periodontitis sites participated in this study. All clinical procedures were approved by the Ethics Committee and informed consent was obtained from each patient. A detailed medical and dental history was obtained from each patient. The experimental protocol which was used to discriminate refractory from successfully treated subjects was the method described in detail by Colombo et al. (3). In brief, subjects received full-mouth scaling and root planing under local anesthesia and instruction in proper home care.

The subjects were monitored at 3-month intervals for one year. If a subject exhibited mean full mouth attachment loss and/or $>3$ sites that exhibited $>2.5 \mathrm{~mm}$ new attachment loss at any of the 3-month visits, he/ she received periodontal surgery accompanied by systemically administered tetracycline. Surgically treated subjects continued to be monitored for a further year. Successfully treated subjects were those who exhibited full-mouth mean attachment level gain and no sites with new attachment loss $>2.5 \mathrm{~mm} 1$ year after either scaling and root planing or surgery with tetracycline. Refractory subjects showed mean full-mouth attachment loss and/or $<3$ sites with new attachment loss $<2.5 \mathrm{~mm}$ after both SRP and periodontal surgery with systemically administered tetracycline. Seventy-three subjects were classified as successfully treated and 27 subjects were classified as refractory. Of the 73 successfully treated subjects 27 subjects were randomly selected as the controls. Samples were randomly obtained from the 
controls. Random samples were taken from 27 chronic refractory periodontitis subjects.

Sample sites were isolated with cotton rolls, supragingival plaque was removed, and one sterile paper point was inserted to the depth of each periodontal pocket sampled. Afterwards, two sequential paper points were placed to the same level to soak up the fluid in the pocket. Each paper point was retained in position for $60 \mathrm{~s}$. Two paper points were then transferred to sterile 2-ml Eppendorf tubes containing VMGA III transport medium (15). All the samples were processed within $2 \mathrm{~h}$. After thoroughly shaking the periodontal sample in a mixer for $60 \mathrm{~s}$ (Vortex, Scientific Industries Inc., Springfield, MA) $1 \mathrm{ml}$ of each sample was used for culture.

The tubes containing samples in VMGA III were preincubated for $30 \mathrm{~min}$ at $37^{\circ} \mathrm{C}$ and shaken vigorously in a vortex mixer (Vortex, Scientific Industries, Inc. Springfield, MA) for $60 \mathrm{~s}$. Serial 10-fold dilutions were made up to $1: 106$ in $1 \%$ sterile peptone water (Bacto Peptone, Difco, Detroit MI, USA). From the serial dilutions, 0.1 $\mathrm{ml}$ was transferred and plated on Brucella (BBL, Becton Dickinson Microbiological Systems, Cockeysville, Md) blood agar plates. The plates were incubated in an anaerobic chamber for $48 \mathrm{~h}$ and the E. faecalis colonies were identified. The purity of the cultures was confirmed by Gram staining, catalase production, colony morphology on blood agar and use of a biochemical identification kit (API 20 Strep, BioMerieux: MarcyI'Etoile, France).

Statistical analysis

The outcome variable was the presence of E. faecalis at the end of periodontal treatment. A subject was classified as either refractory or successfully treated using the criteria described above. Statistical evaluation was performed for descriptive purposes. The exact chisquare test was used to compare the presence of $\mathrm{E}$. faecalis between the two groups. A subject was regarded as positive for $\mathrm{E}$. faecalis if the organism was detected in at least one periodontal pocket. P-values of 0.05 were considered significant.

\section{Results}

Among 100 subjects, only 27 (27\%) had chronic refractory periodontitis. The presence of $\mathrm{E}$. faecalis was evaluated in these 27 subjects of refractory group and in 27 (from 73) of the control group by culture methods. The mean ages in groups 1 and 2 were $41.25 \pm 1.7$ and $42.45 \pm 2.9$, respectively, demonstrating no statistically significant differences between the two groups $(\mathrm{P}=0.13$, $\mathrm{df}=98, \mathrm{t}=1.51$ ).

The difference in the presence of $E$. faecalis in the pockets between the successfully treated subjects (11.1\%) and refractory (51.8\%) subjects by culture methods was statistically significant $(\mathrm{P}<0.05)$.

\section{Discussion}

The present study examined subgingival biofilm samples from patients with refractory periodontitis and successfully treated periodontal patients for the presence of E. faecalis using the culture technique. Consistent with the literature (3-6) we detected a quite high prevalence $(34.9 \%)$ of E. faecalis in the oral samples of all the subjects. Data correlating periodontal infection and E. faecalis colonization are limited.

In general, the prevalence of $\mathrm{E}$. faecalis in the oral cavity seems to be high in individuals with periodontitis $(3,6,11,12)$. Our results showed a significantly higher frequency of $\mathrm{E}$. faecalis in refractory group compared to successfully treated controls $(51.8 \%$ and $11.1 \%$, respectively). Rams et al. (5) detected E. faecalis in only $1 \%$ of early-onset periodontitis and $5.1 \%$ of chronic periodontitis patients using culture methods, whereas Souto et al. (12) reported a much higher prevalence of this species $(80 \%)$ in a large number of subgingival biofilm samples from periodontitis patients. Moreover, significant positive correlations were observed between the presence of $\mathrm{E}$. faecalis and clinical parameters of probing depth, clinical attachment level, bleeding on probing and plaque accumulation.

The higher frequency of $\mathrm{E}$. faecalis in patients with periodontitis suggests that the establishment of this organism seems to be favored by the presence of a complex subgingival microbiota and periodontal inflammation (16). One could argue that periodontal pockets present a very rich environment for the colonization of $E$. faecalis. The complex and diverse microbiota along with the persistent inflammatory process may provide a wide range of nutrients and binding sites for the establishment of this microorganism (16). In a recent study, Johnson et al. (17) showed coaggregation interactions between E. Faecalis and F. nucleatum isolates from persistent apical periodontitis. Fusobacterium spp., described as members of the "orange complex", have been shown to increase in frequency and levels in sites with deep periodontal pockets, loss of periodontal attachment and bleeding on probing (16). Thus, high proportions of Fusobacterium may favor colonization by E. faecalis in the periodontal environment. Likewise, Colombo et al. (3) reported high prevalence and levels of periodontal pathogens, as well as of E. faecalis, in subgingival biofilm samples of chronic periodontitis patients. On the other hand, the positive associations observed between clinical measurements of periodontal destruction and inflammation (pocket depth, clinical attachment level, bleeding on probing and plaque index) and the presence of E. faecalis may suggest that this species also plays a role in the severity and/or progression of periodontitis. Indeed, oral enterococci produce virulence factors of possible relevance to the pathogenesis of periodontitis, including aggregation substances, surface adhesins, 
lipoteichoic acid, extracellular superoxide production, lytic enzymes such as gelatinase, hyaluronidase and elastase, the toxin cytolysin, and haemolysins capable of inducing neutrophil dysfunction (2). Each of them may be associated with various stages of periodontal infection. Moreover, E. faecalis may enhance pathogenicity in mixed infections with anaerobic bacteria (18) and is capable of inducing experimental apical periodontitis in combination with other oral bacteria (19).

The diagnosis of "refractory" periodontitis should only be made in patients who satisfactorily comply with recommended oral hygiene procedures and follow a rigorous program of periodontal maintenance (14). These individuals are referred to as "refractory periodontitis patients." Prominent periodontal pathogens such as P. gingivalis, T. forsythia, H. nucleatran, P. micros, E. corrodens, and Streptococcus intermedius have been found to be elevated in patients who do not respond to treatment. Often, patients identified as refractory to treatment have other factors, particularly smoking, which may contribute to the disease process. In a recent study, Gonçalves et al. (11) reported that E. faecalis is more commonly found in the subgingival microbiota of HIV-infected than in nonHIV-infected subjects with periodontitis. Since there have been reports about the role of immune system deficiency in the etiology of refractory periodontitis, there seems to be a relationship between immunodeficiency, E. faecalis and refractory periodontitis.

Detection of E. faecalis in refractory sites seemed to have a role in the pathogenesis of refractory periodontitis. The limitations of this study were:

1. PCR was not used to detect E. faecalis.

2. Sample size was small.

3. No sampling was carried out at baseline.

\section{Conclusion}

This study tried to detect E. faecalis in refractory sites, which is probably involved in the pathogenesis of refractory periodontitis. Sound knowledge about the pathogen and its role in the pathogenesis of refractory infections helps develop effective strategies in treating the infections. Patients whose disease is clearly refractory are candidates for bacterial culturing and antibiotic therapy in conjunction with additional mechanical therapy.

\section{References}

1. Mundy LM, Sahm DF, Gilmore M. Relationships between enterococcal virulence and antimicrobial resistance. Clin Microbiol Rev. 2000;13:513-22.

2. Kayaoglu G, Ørstavik D. Virulence factors of Enterococcus faecalis: relationship to endodontic disease. Crit Rev Oral Biol Med. 2004; 15:308-20.

3. Colombo AP, Teles RP, Torres MC, Souto R, Rosalém WJ, Mendes $\mathrm{MC}$, et al. Subgingival microbiota of Brazilian subjects with untreated chronic periodontitis. J Periodontol. 2002;73:360-9.
4. Gold OG, Jordan HV, van Houte J. The prevalence of enterococci in the human mouth and their pathogenicity in animal models. Arch Oral Biol. 1975;20:473-7.

5. Rams TE, Feik D, Young V, Hammond BF, Slots J. Enterococci in human periodontitis. Oral Microbiol Immunol. 1992;7:249-52.

6. Sedgley CM, Lennan SL, Clewell DB. Prevalence, phenotype and genotype of oral enterococci. Oral Microbiol Immunol. 2004;19:95101.

7. Sedgley C, Buck G, Appelbe O. Prevalence of Enterococcus faecalis at multiple oral sites in endodontic patients using culture and PCR. J Endod. 2006;32:104-9.

8. Rôças IN, Siqueira JF Jr, Santos KR. Association of Enterococcus faecalis with different forms of periradicular diseases. J Endod. 2004;30:315-20.

9. Sundqvist G, Figdor D, Persson S, Sjögren U. Microbiologic analysis of teeth with failed endodontic treatment and the outcome of conservative re-treatment. Oral Surg Oral Med Oral Pathol Oral Radiol Endod. 1998;85:86-93.

10. Slots J, Feik D, Rams TE. Prevalence and antimicrobial susceptibility of Enterobacteriaceae, Pseudomonadaceae and Acinetobacter in human periodontitis. Oral Microbiol Immunol. 1990;5:149-54.

11. Gonçalves Lde S, Soares Ferreira SM, Souza CO, Souto R, Colombo AP. Clinical and microbiological profiles of human immunodeficiency virus (HIV)-seropositive Brazilians undergoing highly active antiretroviral therapy and HIV-seronegative Brazilians with chronic periodontitis. J Periodontol. 2007;78:87-96.

12. Souto R, Colombo AP. Prevalence of Enterococcus faecalis in subgingival biofilm and saliva of subjects with chronic periodontal infection. Arch Oral Biol. 2008;53:155-60.

13. Tanriverdi F, Esener T, Erganiş O, Belli S. An in vitro test model for investigation of disinfection of dentinal tubules infected with Enterococcus faecalis. Braz Dent J. 1997;8:67-72.

14. Parameter on "refractory" periodontitis. American Academy of Periodontology. J Periodontol. 2000;71:859-60.

15. Dahlén G, Pipattanagovit P, Rosling B, Möller AJ. A comparison of two transport media for saliva and subgingival samples. Oral Microbiol Immunol. 1993;8:375-82.

16. Socransky SS, Haffajee AD, Cugini MA, Smith C, Kent RL Jr. Microbial complexes in subgingival plaque. J Clin Periodontol. 1998;25:134-44.

17. Johnson EM, Flannagan SE, Sedgley CM. Coaggregation interactions between oral and endodontic Enterococcus faecalis and bacterial species isolated from persistent apical periodontitis. J Endod. 2006;32:946-50

18. Brook I. Effect of Streptococcus faecalis on the growth of Bacteroides species and anaerobic cocci in mixed infection. Surgery. 1988;103:107-10

19. Fabricius L, Dahlén G, Holm SE, Möller AJ. Influence of combinations of oral bacteria on periapical tissues of monkeys. Scand J Dent Res. 1982;90:200-6. 